\title{
A previously undescribed variant of the confluence of sinuses
}

\author{
G.G. Tardieu1 ${ }^{1}$, R.J. Oskouian², M. Loukas ${ }^{1}$, R.S. Tubbs 3 \\ ${ }^{1}$ Department of Anatomical Sciences, St. George's University, Grenada, West Indies \\ ${ }^{2}$ Swedish Neuroscience Institute, Swedish Medical Centre, Seattle, WA, United States \\ ${ }^{3}$ Seattle Science Foundation, Seattle, Washington, United States
}

[Received: 7 July 2016; Accepted: 24 August 2016]

\begin{abstract}
An 8-year-old female with a history of chronic headaches and uncertain papilloedema was found to have a variant of the posterior intracranial dural venous sinuses on magnetic resonance imaging assessment of the brain. Magnetic resonance venography included in the imaging revealed a circular formation of the confluence of sinuses and absent right-sided transverse sinus. The confluence of sinuses is a highly variable structure; however, to the authors' knowledge, a circular confluence of sinuses variant has not been reported in the literature. (Folia Morphol 2017; 76, 2: 316-318)
\end{abstract}

Key words: intracranial, anomalies, dura mater, anatomy

\section{INTRODUCTION}

The confluence of sinuses (CS) also known as the torcular Herophili is an important junction in the system of the dural venous sinuses, which is greatly variable. It is known historically to connect the superior sagittal sinus (SSS), the straight sinus (StS), the occipital sinus (OS), and the transverse sinuses (TSs) $[3,4,7]$. Generally, the SSS, OS and the StS flow toward the CS, while the two TSs flow away from the CS. Depending largely on (1) the individual sinus diameters, (2) the particular anomaly of the CS, and (3) the presence of intraluminal septations present in the region of the CS, the direction of flow can vary from patient to patient.

There are many reported variations of the dural venous sinuses in the area of the CS $[1,3,4,6]$. Herein, we report, to our knowledge, a previously unreported variant of the $\mathrm{CS}$.

\section{CASE REPORT}

During magnetic resonance imaging (MRI) evaluation of the brain in an 8-year-old female patient, a variant of the posterior intracranial dural venous sinuses was observed. The child had a previous history of chronic headache and questionable papilloedema on physical examination. Her head circumference was at the $50 \%$ for age. She was slightly developmentally delayed. Brain imaging had been requested for evaluation of potential pseudotumour cerebri. MRI revealed no intracranial pathology, such as hydrocephalus. Imaging included magnetic resonance venography and this demonstrated a circular formation of the CS and absent right-sided TS (Figs. 1, 2).

\section{DISCUSSION}

In the human embryo, the initial organisation for capillary drainage begins with a main head vein in the area of the midbrain that travels beside the neural tube caudally. The main head vein drains blood from the network of capillaries, which is the origin of venous drainage from the neural tube. Arising from the capillaries are anastomosing venous loops,

Address for correspondence: Dr. G.G. Tardieu, Department of Anatomical Sciences, St. George's University, True Blue, Grenada, West Indies, tel: 908-800-3846, e-mail: gtardieu@sgu.edu 


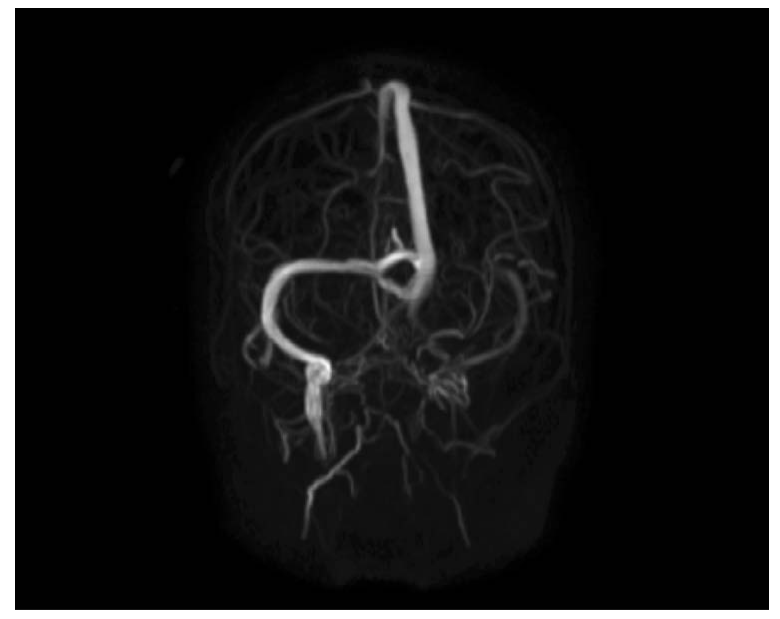

Figure 1. Magnetic resonance venography (posterior view) of patient described herein noting the circular formation of the confluence of sinuses. Also note the absence of the right transverse sinus.

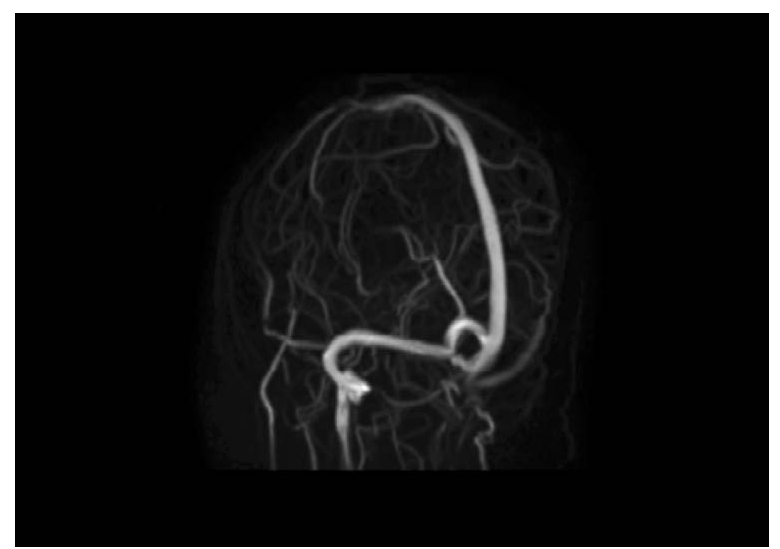

Figure 2. Magnetic resonance venography (left lateral view) of patient seen in Figure 1 noting the deep cerebral drainage into the upper component of the circular confluence of sinuses.

which drain into the following three plexuses: (1) the anterior dural plexus, (2) the middle dural plexus and (3) the posterior dural plexus. As forebrain growth progresses, the anterior plexus is shifted caudally with a part of its anterior network remaining. This part becomes the sagittal plexus and in the midline, the SSS develops from the sagittal plexus. The StS is formed from a branch of the anterior plexus. The anterior plexus then joins with the middle plexus, as the embryo develops, and drains caudalward becoming less evolved than its earlier form, creating the junction where the SSS, the StS, the OS and the two TSs meet. This results in the formation of the primitive CS, the last remnants of the anterior and middle dural plexuses $[2,6,8]$.
Kobayashi et al. [6] described 9 different variations of the CS, with type 1 being the most frequently observed, which correlates with the historical definition of the CS [4].

- Type 1 - The SSS, StS and the two TSs intersect at one point (32.7\% of patients).

- Type 2 - Bilateral TSs are branches of the StS; Type $2 \mathrm{a}$ - The SSS connects to the right TS $(31.5 \%$ of patients); Type $2 b$ - The SSS connects to the left TS $(6.2 \%$ of patients).

- Type 3 - Bilateral TSs are branches of both the SSS and the StS (18.9\% of patients).

- Type 4 - Bilateral TSs are branches of the SSS; Type $4 \mathrm{a}$ - The StS connects to the right TS (3.1\% of patients); Type $4 \mathrm{~b}$ - The StS connects to the left TS (4.4\% of patients).

- Type $5 \mathrm{a}$ - The StS connects to the OS $(0.5 \%$ of patients); Type $5 b$ - The StS connects with the OS as well as with other veins by small anastomotic veins $(0.4 \%$ of patients).

- Type 6 a - A defective left TS ( $0.4 \%$ of patients); Type $6 \mathrm{~b}-\mathrm{A}$ defective right TS ( $0.9 \%$ of patients).

- Type 7 - The SSS drains into the StS sinus and the left TS, and the StS branches into bilateral TSs $(0.2 \%$ of patients).

- Type 8 - The StS drains into the SSS and the left TS, and the SSS branches into bilateral TSs $(0.4 \%$ of patients).

- Type 9 - The StS drains into the left TS and OS, the SSS branches into the right TS and OS, and the SSS connects with the StS only by the small anastomotic veins ( $0.4 \%$ of patients).

The connection between the right and left TSs was categorised into four types as follows:

- Type A - wide connection ( $88.1 \%$ of patients);

- Type B - narrow connection (10.2\% of patients);

- Type C - no connection ( $0.4 \%$ of patients);

- Type D - single TS (1.3\% of patients).

Though type 1 was observed to be the most common, according to Kobayashi et al. [6], it might not be the type existing in the majority of the population. As a matter of fact, the SSS drainage is commonly uneven, with drainage primarily or entirely into the right TS, which occurs three times more frequently than on the left. Park et al. [7] observed that in 51.6\% of patients, the SSS drained into the right TS, and in $35.5 \%$ of patients, their right TS was dominant obtaining a diameter of greater than 1.5 times that of the left TS. In only $3.2 \%$ of patients the left TS was dominant. Contrary to this, Curé et al. [3] reported 
that the StS branches into the left TS three times more often than the right. In most of the population, the right and the left TSs were proportional $(58.1 \%$ of patients) [7]. Abnormal development of one of the TSs was observed in $5 \%$ of cases at autopsy with three being on the left and seven on the right, and the SSS connecting to the opposite TS which was developed [5]. Diversity was also observed in the SSS with drainage to the centre of the CS in $58.1 \%$ (18 of 31) of patients, and in $35.5 \%$ (11) drainage into the left TS [7].

The presence of one versus two TSs, and the communication between the two in the region of the CS can determine, somewhat, the risk and degree of cerebral oedema, especially if there is obstruction of the TS or sigmoid sinus during surgery. In agreement with Ishizaka [4], it is thought that the variant that has two TSs communicating widely between them results in the smallest risk of cerebral oedema. A rare variant of a circular CS as seen in our case could lead to increased risk of cerebral oedema due to decreased available area for drainage from the dural venous sinuses, such as the SSS and the StS which drained into the circular CS. The other known anomaly in our patient was a unilateral developed left TS with the right TS being absent. As postulated in Ishizaka [4], this may further increase the risk of venous sinus congestion and cerebral oedema and might have contributed to the raised intracranial pressure as seen in our patient.

\section{CONCLUSIONS}

The CS is a well-encountered structure in brain surgery and interventional procedures in posterior fossa lesions where the venous route is used. Thus, it is of critical importance to be able to recognise each dural sinus pre- and intra-operatively, with special attention to any anomalies present, to prevent iatrogenic injury to the dural sinuses during craniotomy and other procedures involving the posterior cranial fossa. The rare variation of a circular CS in our patient could be classified as a new type 10 variation to add to the nine types previously outlined by Kobayashi et al. [6].

\section{REFERENCES}

1. Bisaria KK. Anatomic variations of venous sinuses in the region of the torcular Herophili. J Neurosurg. 1985; 62(1): 90-95, doi: 10.3171/jns.1985.62.1.0090, indexed in Pubmed: 3964860.

2. Butler $\mathrm{H}$. The development of certain human dural venous sinuses. J Anat. 1957; 91(4): 510-526, indexed in Pubmed: 13475149.

3. Curé J, Tassel P, Smith M. Normal and variant anatomy of the dural venous sinuses. Seminars Ultrasound, CT MRI. 1994; 15(6): 499-519, doi: 10.1016/s08872171(05)80019-8.

4. Ishizaka H. Anatomical study of the torcular herophili. Neurol Med Chir. 1985; 25(11): 873-880, doi: 10.2176/ nmc.25.873.

5. Kaplan H, Browder J, Knightly J, et al. Variations of the cerebral dural sinuses at the torcular herophili. Am J Surg. 1972; 124(4): 456-461, doi: 10.1016/00029610(72)90066-9.

6. Kobayashi K, Matsui O, Suzuki M, et al. Anatomical study of the confluence of the sinuses with contrast-enhanced magnetic resonance venography. Neuroradiology. 2006; 48(5): 307-311, doi: 10.1007/s00234-006-0065-4, indexed in Pubmed: 16575556.

7. Park HKi, Bae HG, Choi SK, et al. Morphological study of sinus flow in the confluence of sinuses. Clin Anat. 2008; 21(4): 294-300, doi: 10.1002/ca.20620, indexed in Pubmed: 18428995.

8. Streeter $\mathrm{G}$. The development of the venous sinuses of the dura mater in the human embryo. Am J Anat. 1915; 18(2): 145-178, doi: 10.1002/aja.1000180202. 